\title{
Orthodontic management in patients with haemophilia. About two clinical cases
}

\author{
Gerardo Gómez-Moreno ${ }^{1}$, María-Elena Cañete-Sánchez ${ }^{2}$, Javier Guardia ${ }^{3}$, Tania Castillo-Naveros ${ }^{4}$, José- \\ Luis Calvo-Guirado ${ }^{5}$
}

\author{
${ }^{1}$ Professor Responsible of Pharmacological Interactions in Dentistry. Faculty of Dentistry. University of Granada \\ ${ }^{2}$ Degree in Dentistry. Master of Orthodontics. Universidad Complutense de Madrid \\ ${ }^{3}$ Doctor in Dentistry. Collaborator in Pharmacological Interactions in Dentistry. Faculty of Dentistry. University of Granada \\ ${ }^{4}$ Collaborator in Pharmacological Interactions in Dentistry. Faculty of Dentistry. University of Granada \\ ${ }^{5}$ Senior Lecturer, Adult Integrated Dentistry Clinic and Director of Implantology Masters Program, Faculty of Medicine and \\ Dentistry, University of Murcia
}

Correspondence:

Campus Universitario Cartuja $s / n$

18071 Granada. Spain

ggomez@ugr.es

Received: 04/02/2009 Accepted: 12/09/2009
Gómez-Moreno G, Cañete-Sánchez ME, Guardia J, Castillo-Naveros T, Calvo-Guirado JL. Orthodontic management in patients with haemophilia. About two clinical cases. Med Oral Patol Oral Cir Bucal. 2010 May 1;15 (3):e463-6.

http://www.medicinaoral.com/medoralfree01/v15i3/medoralv15i3p463.pdf

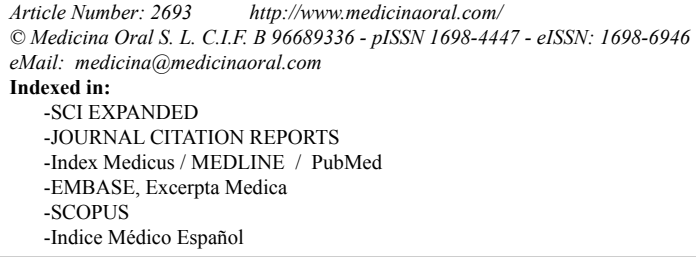

\begin{abstract}
The most common congenital bleeding disorder is haemophilia. It is a pathology inherited and caused by a defective or an absence of the coagulation factors, so that haemophiliacs cannot form an efficient clot. These patients have been treated with fear in the dental profession without having achieved the goals and ideals most appropriate according to the requirements of each case, which is demonstrated in the little existing literature available. However, they are currently treated as healthy orthodontic patients thanks to the advances in orthodontics. We present the cases of two brothers with mild classical haemophilia, who were treated by Mc Namara's disjunctor. The emphasis is on importance of interconsultation with the haematologist, prevention and oral hygiene, and that of the beneficial effect of orthodontic.
\end{abstract}

Key words: Orthodontics, haemophilia.

\section{Introduction}

The dental management of patients with haemophilia has been an object of interest to dental professionals in recent decades. The orthodontic treatment of these patients has been neglected for years for fear of bleeding, which involved complications in the occlusal, dental and periodontal state of the same. These patients have higher risk of complications during orthodontic treat- ment, but they can get a successful outcome to the extent that they present a proper control of their disease (1-3).

\section{Haemophilia}

It is the most common congenital bleeding disorder (4). Haemophilia is a congenital Haematological condition inherited and caused by a deficient activity or absence of clotting factors that are essential for normal haemos- 
tasis. Thus, haemophiliacs cannot efficiently form a clot (5). The severity of haemophilia depends on the amount of this coagulation factor. The normal concentrations of clotting factor are between $50 \%$ and $150 \%$ of average value and the minimum level of a factor for adequate haemostasis is $25 \%(2)$.

Haemophilia is classified as mild, when the decrease of factor VIII is $5-25 \%$ of normal; moderate, when the decrease is $1-5 \%$; and severe when the decrease is less than $1 \%$. When levels of clotting factor is found below $1 \%$ it can cause spontaneous bleeding (2). There are different types of haemophilia $(2,4)$ :

-Haemophilia A, or classic or deficit of factor VIII: this kind of haemophilia is the most frequent and presents in 1 in every 10,000 people. Approximately 80\% of affected have this type of.

-Haemophilia B, or Christmas disease or Factor IX deficiency: is present in 15 persons per million (3). Represents $15 \%$ of those affected. These patients did not respond to desmopressin (DDAVP).

-Haemophilia C, or factor X deficiency: it's a mild and less frequent form of the disease.

The haemophiliac patient usually present a normal analytical bleeding time, platelet recount and aggregation. This process is sufficient to produce haemostasis in small puncture wounds and lacerations. However, there appears an increased prothrombin times and / or thromboplastin; therefore, the clotting mechanism does not fulfill its normal function in these patients $(2,6)$.

A thorough medical and dental history and a complete review are essential components in the management of haemophiliac patients. It is of vital importance to know the type and severity of the disease, the presence or not of inhibitor agent, the current medications, family history of the condition, previous hospitalizations, transfusions, other diseases (including hepatitis and HIV by replacement therapy) and previous dental treatments (7). It is necessary to know which are the invasive dental procedures that requiere a propiate preoperative coverage of the haemophiliac patient, because there may be an increased risk of significant bleeding. Therefore, the dental management of these patients requires collaboration and integration of the haematologist and the professional in the oral cavity $(4,8)$.

\section{Orthodontics}

In patients with haemophilia the best dental treatment is preventive. A preventive and interceptive orthodontic treatment, when possible, can solve a prior mloclusive pathology much more severe. It is therefore very important an early diagnosis and early detection of occlusal disarmonía as it can eliminate orthodontic problems in the future (9).

Years ago, orthodontics was avoided in patients with bleeding disorders. The ignorance on the part of professionals, lack of coordination between the haematolo- gist and the dentist and the already outdated Multiband orthodontic techniques were the cause. Currently, this does not happen because the bands are prefabricated and can be replaced by tubes and the technique is not Multiband but multibrackets, bonded directly or indirectly, thereby minimizing the possible damage to the gums. The attitude has changed and patients with haematological disorders are now treated in the same way as the normal orthodontic patients; in this sense, the criteria for the indication and duration of orthodontic treatment are the same $(1,6,9)$

\section{Discussion}

We present the cases of two brothers, 8 and 10 years of age with mild classic haemophilia diagnosed at the age of 4 and 6 years respectively. Both had maxillary basal compression that was more pronounced in the older brother. Such compression generated unilateral posterior crossbite and mandibular deviation of functional cause. This type of malocclusion is not uncommon and orthodontic treatment is recomended as it must balance the sizes of both maxillary to enable that the bone development is aproppiate.

The treatment prescribed after the study of orthodontics was a first phase by disjunction to correct the transverse discrepancies, and then reassess both cases. The device chosen for this was the Mc Namara disjunctor.

To assess the risk of orthodontic treatment (3) is convenient to divide the treatment procedures into two groups:

1. Invasive procedures that can generate bleeding:

Extractions, surgical manipulation of periodontal tissues, including an exhibition of a tooth, placement of bands with a subgingival extention and orthognathic surgery.

2. Non-invasive procedures with minimal risk of bleeding: making impressions, placement of fixed appliances with direct bonding, stripping, routine adjustment of equipment and insertion and adjustment of removable appliances.

If orthodontic treatment is in the first group, the patient will require a preoperative evaluation and postoperative follow-up. This is not necessary for those patients who are in the second group of the classification.

The Mc Namara's disjunctor belongs to the second group of the classification. However, it was consulted with the haematologist who sent a report indicating that only in processes such as extractions and surgeries a preoperative coverage was required.

We proceeded to take modeling for the design of the disjunctor covering the edges of the trays with wax to prevent soft tissue damage. A week later a McNamara disjunctor was fixed to each brother and the parents were explained the activations of the appliance and the importance of oral health in the bearers of fixed appliances. 
There was attached an instruction sheet and a list aimed to daily record the number of times the screw expander was turned. The protocol of activations was the conventionally used in such apparatus for achieving disjunction of suturing palate.

The orthodontic braces are retentive in nature, which together with the accumulation of plaque in the margin of the gum can contribute to the incidence and severity of gingival inflammation (10). The best way to prevent gum bleeding is establishing and maintaining healthy gums through excellent oral hygiene and stimulation of tissues. It has been observed that the state of oral hygiene is often worse in the haemophiliac patients (11); which speaks in favor of the beneficial effects of use of oral hygiene programs in conjunction with the Orthodontic treatment (10).

14 days later the disjunction was stopped in the younger brother and 18 days later in the older brother. Both got the overcorrective expansion desired, as is standard in handling this type of equipment. The screw was closed and the period of detention was started, that would last approximately six months. We began monthly controls to check the state of the disjunctor, oral hygiene and closing the expansion screw in both brothers. The younger brother was deteriorating his state of hygiene progressively without heeding our preventive advices neither the oral hygiene. Finally, in the control of the fourth month, a suspected ulcer below the disjunctor due to the inflammation of the edges of it, a decision was made to extract the device before the time was ideally indicated. Before extracting the disjunctor we valuated the risk of bleeding (12) with a prior determination by the haematologist of the activity Factor VIII $(13,14)$, which decided that four hours before going to the dental hospital, at hospital level he was administered for 20 minutes $0.3 \mathrm{mg} / \mathrm{kg}$ of intravenous desmopressin. Confirming the suspicions, large swelling and an ulcerated area below the acrylic of the disjunctor was found (Fig. 1).

Bleeding after extractinon lasted about ten minutes, and the patient was kept under observation an hour, after which there was no bleeding, therefore oral tranexamic acid was not prescribed. After a week a removable Quad-Helix was placed so as not to lose the expansion achieved and 15 days later, all palatal mucosa recovered to normal.

The older brother did complete the retention period. Due to the perception of some clinical mobility of the device and because the patient was set to begin second phase of replacement teeth, it was suspected that at extracting the device it could traction from some temporal teeth reabsorbed and near exfoliation.

Therefore, this factor was consulted with the haematologist and the visit was delayed.

Two weeks later, and four hours before going to the

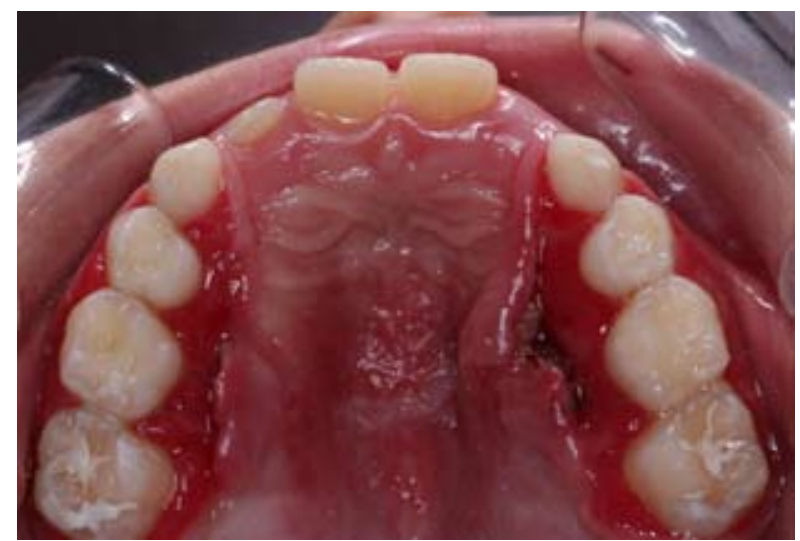

Fig. 1. Great inflammation and ulceration after uncemented disjunctor.

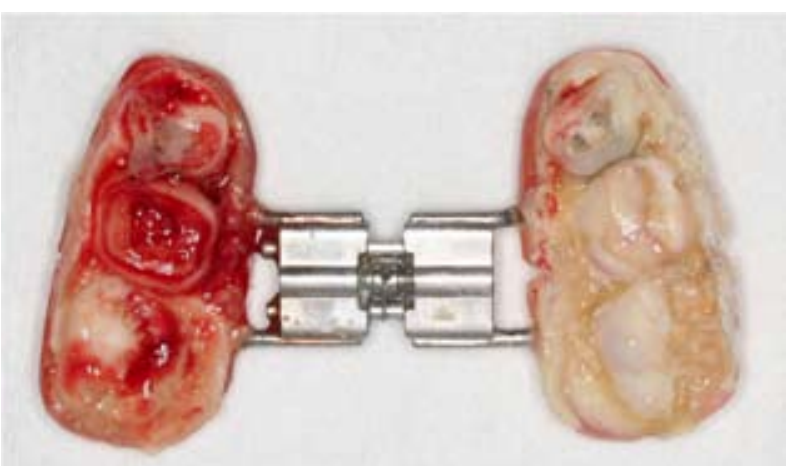

Fig. 2. McNamara disjunctor. It can be observed 54, 64 and 65 are reabsorbed and attached to the acrylic.

dental consultation, at a hospital level he was administered during 20 minutes $0.3 \mathrm{mg} / \mathrm{kg}$ of intravenous desmopressin, and we proceeded to extract the disjunctor . Three primary molars normally reabsorbed (as his replacement) during the retention period were extracted with the disjunctor (Fig. 2). Bleeding and gingival status were normal so it was placed so maintenance Quad-helix in that same visit. Once the disjunctor was removed and following the directions of the Service of Haematology, the patient was prescribed $30 \mathrm{mg} / \mathrm{kg}$ oral tranexamic acid, continued in his home four times a day, for that day $(2,15)$.

The desired disjunction and correction of the maxilliary compression was achieved in both brothers and, therefore, the treatment was successful.

\section{Conclusion}

Haemophilia nowadays is not an absolute contraindication to orthodontic treatment.

The coordination and communication of haematologistprofessional in the oral cavity is essential. Thanks to advances in orthodontics there is no contraindication 
per se in haemophiliac patients, and the decision to carry out treatment is made with the same criteria as any other patient. It must be emphasized in the awareness of the importance of oral hygiene and the initial prevention due to the numerous benefits they can bring, moreover avoiding risks. Finally, note that there is little literature and standardized and consensuated protocols for the management of haemophilia patients carriers of orthodontic.

\section{References}

1. Azhar S, Yazdanie N, Muhammad N. Periodontal status and IOTN interventions among young hemophiliacs. Haemophilia. 2006;12:401-4.

2. Gómez-Moreno G, Cutando-Soriano A, Arana C, Scully C. Hereditary blood coagulation disorders: management and dental treatment. J Dent Res. 2005;84:978-85.

3. Van Venrooy JR, Proffit WR. Orthodontic care for medically compromised patients: possibilities and limitations. J Am Dent Assoc. 1985;111:262-6.

4. Jover-Cerveró A, Poveda Roda R, Bagán JV, Jiménez Soriano Y. Dental treatment of patients with coagulation factor alterations: an update. Med Oral Patol Oral Cir Bucal. 2007;12:E380-7.

5. Katz JO, Terezhalmy GT. Dental management of the patient with hemophilia. Oral Surg Oral Med Oral Pathol. 1988;66:139-44.

6. Evans BE, Aledort LM. Hemophilia and dental treatment. J Am Dent Assoc. 1978;96:827-34

7. Vangelisti R, Pagnacco O, Ristagno G, Ruggeri M, Tosetto A, Castaman G, et al. Prevention of hemorrhage and dental treatment of patients with congenital or acquired coagulopathies. Minerva Stomatol. 1997;46:621-6.

8. Zanon E, Martinelli F, Bacci C, Zerbinati P, Girolami A. Proposal of a standard approach to dental extraction in haemophilia patients. A case-control study with good results. Haemophilia. 2000;6:533-6. 9. Grossman RC. Orthodontics and dentistry for the hemophilic patient. Am J Orthod. 1975;68:391-403.

10. Yeung SC, Howell S, Fahey P. Oral hygiene program for orthodontic patients. Am J Orthod Dentofacial Orthop. 1989;96:208-13.

11. Mielnik-Błaszczak M. Evaluation of dentition status and oral hygiene in Polish children and adolescents with congenital haemorrhagic diatheses. Int J Paediatr Dent. 1999;9:99-103.

12. White GC 2nd, Blatt PM, McMillan CW, Webster WP, Lesesne HR, Roberts HR. Medical complications of hemophilia. South Med J. 1980;73:155-60.

13. Redding SW, Stiegler KE. Dental management of the classic hemophiliac with inhibitors. Oral Surg Oral Med Oral Pathol. 1983;56:145-8.

14. Johnson WT, Leary JM. Management of dental patients with bleeding disorders: review and update. Oral Surg Oral Med Oral Pathol. 1988;66:297-303.

15. Goss AN. The dental management of medically compromised patients. Int Dent J. 1984;34:227-31. 